\title{
Theory for weakly polydisperse cytoskeleton filaments
}

\author{
Vadim Warshavsky and Marcelo Marucho* \\ Department of Physics and Astronomy, \\ University of Texas at San Antonio, TX 78249, USA
}

The ability of actins and tubulins to change dynamically from alterations in the number density of actins/tubulin, number density and type of binding agents, and electrolyte concentration is crucial for eukaryotic cells to regulate their cytoskeleton conformations in different cellular compartments. Conventional approaches on biopolymers solution break down for cytoskeleton filaments because they entail several approximations to treat their polyelectrolyte and mechanical properties. In this article, we introduce a novel density functional theory for polydisperse, semiflexible cytoskeleton filaments. The approach accounts for the equilibrium polymerization kinetics, length, and orientation filament distributions, as well as the electrostatic interaction between filaments and the electrolyte. This is essential for cytoskeleton polymerization in different cell compartments generating filaments of different lengths, sometimes long enough to become semiflexible. We characterized the thermodynamics properties of actin filaments in electrolyte aqueous solutions. We calculated the free energy, pressure, chemical potential, and second virial coefficient for each filament conformation. We also calculated the phase diagram of actin filaments solution and compared it with available experimental data.

\section{INTRODUCTION}

Eukaryotic cells can dynamically regulate their own biological environment and the polyelectrolyte and mechanical properties of cytoskeleton filaments to achieve specific biological functions as diverse as directional growth, shape, division, plasticity, and migration.[1] These selforganization behavior, yet poorly understood, has been observed experimentally. The increase in the number density of G-actins/tubulin and electrolyte concentration can lead to conformation transformations from orientation disorder (isotropic) to an orientation ordered (nematic) phase,

\footnotetext{
*Electronic address: marcelo.marucho@utsa.edu
} 
as well as increasing the filaments average length. Additionally, the growth of the number density of binding agents, such as divalent ions or linker proteins can yield bundling or network conformations. Indeed, an increasing number of microscopy images visualized with micro-scale resolutions in cells have been published on actin filament and MT structures. These images provide valuable information on the distribution and type of cytoskeleton conformations in cells. Nowadays, confocal microscopies can also capture dynamic conformation changes from their movies. However, they usually provide an incomplete understanding of the impact of the cell type and compartment conditions on conformational dynamics, self-organization, and stability, which vary depending on the cell type and location, gender, age, and inheritance conditions. When accounting for all of these features, one can formulate a quantitative description of their conformation properties. This understanding might elucidate the biophysical principles underlying fundamental biological functions of eukaryotic cells in normal and pathological conditions.

A substantial amount of research has been done in the field to study the isotropic to nematic phase transformation in macromolecules solution. Conventional understanding of the properties of these polyelectrolytes is based on monodisperse, mean-field theories, and rod-like cylindrical filament models. These methods break down for cytoskeleton filaments because they entail several approximations to treat the inter-filament interactions, electrolytes, and molecular filament structures.

Onsager [28] showed that the expansion of the free energy functional up to the second virial coefficient for electrically charged long rods provided accurate results for the coexisting isotropic and nematic phases. In a nematic phase, the free energy was expressed as a functional of the angular distribution function of rods that minimizes the free energy functional. Onsager introduced a trial function for the angular distribution function depending on a single parameter to simplify calculations. Subsequently, a variety of versions of the Onsager's theory were proposed $[11,12]$. For instance, Odijk proposed a trial function of a Gaussian type for the angular distribution, which was less accurate than the Onsager's one, but it further simplified the theory [20].

Corrections were made in the orientational part of Onsager's free energy to treat semiflexible macromolecules with sizes larger than the persistence length $[9,10]$. Later, some approximations were developed to patch the orientational free energies for rigid and semiflexible rods [20, 27].

Sluckin [19] generalized the Onsager's theory to account the polydispersity of rigid rods. In this theory, the size distribution function was considered to have the same Gaussian form both in isotropic and nematic phases. Additionally, Odijk's anzatz was generalized to the polydisperse 
case. However, the nematic phase onset was formed by rods with lengths larger than the average length of the size distribution in the isotropic phase. Different modifications of the method were proposed [22-24]. In particular, the input size asymmetric distribution functions in the form of Schulz and log-normal distributions were considered.

Other approaches were introduced for the isotropic-nematic phase transformation in amphiphilic micellar suspensions[32]. The size distribution function of micellar macromolecules was not an input value, but rather obtained from special micella equilibrium conditions of different sizes. The characteristic input in this study was the standard chemical potential of amphiphilic micellas, which governed the size-angular distribution functions. It was found that the micella average lengths in the coexisting isotropic and nematic phases were different, with larger micella sizes in the nematic phase.

In this work, we introduce a theory to account for the length and orientation filament distributions, semiflexibility of filaments, and an charged inter-filament potential interaction model to characterize the thermodynamics properties of cytoskeleton filaments in electrolyte aqueous solutions. The formulation has been able to elucidate the set of parameters governing the phase behavior diagram. This approach is essential for G-actin polymerization in different cell compartments generating filaments of different lengths, sometimes long enough to become semiflexible. We followed the experimental conditions on the actin filaments solution, where the transition in the isotropic-nematic phase diagram was due to the change in the G-actin concentration. Additionally, the average size of filaments in those experiments was kept fixed by changing the gelsolin proteins concentration $[14,15]$. We obtained the size distribution function from the special equilibrium conditions (see Eq.27), whereas we used Sluckin's trial function for the angular distribution function in nematic phase. We introduced an anzatz for the standard chemical potential of actin filaments that results in the asymmetric Schulz's distribution function for actin filaments size. This distribution agrees with those used in light scattering experiments on actin filaments[14]. We have accounted for the filament semiflexibility by the generalization of the formula for orientational free energy introduced in Ref.[20] for polydisperse systems. Finally, we have calculated and plotted the isotropic-nematic phase diagrams for some values of normalized standard deviation of Schulz's size distribution, the persistence length of actin filaments and the concentration of monovalent ions in electrolyte solution. We have compared the obtained results with available experimental data [15].

The paper is organized as follows. The method is described in part II, the numerical results 
are given in part III, the discussion is provided in part IV, the details of the calculations are presented in Appendix.

\section{THEORY}

We consider a solution of actin filaments, each of them has the length $L=l_{m} \nu$, where $l_{m}$ is a length of a monomer unit, $\nu$ a number of monomer units in a filament (a size of filament). Each filament has its own direction in 3D space, which is characterized by a body-angle $\omega$ accounted from the chosen nematic director.

We introduce the size-angular density distribution function of filaments $\rho_{\nu}(\omega)$ in the following form

$$
\rho_{\nu}(\omega)=\rho n_{\nu} \eta_{\nu}(\omega)
$$

where $\rho=N / V$ is a total density of filaments ( $N$ is a total number of filaments of any size, $V$ the volume of a system), $n_{\nu}$ a size distribution function and $\eta_{\nu}(\omega)$ an angular distribution function, which is also dependent on size $\nu$.

The normalization conditions for size- and angular- distribution functions $n_{\nu}$ and $\eta_{\nu}(\omega)$ are

$$
\begin{gathered}
\sum_{\nu \geq 1} n_{\nu}=1, \\
\int \eta_{\nu}(\omega) d \omega=1,
\end{gathered}
$$

where the summation in Eq.(2) is performed over all filament sizes $\nu$, the integration in Eq.(3) over the whole body angle.

The size distribution function $n_{\nu}$ is characterized by the average size (the average degree of polymerization) of filaments $\langle\nu\rangle$ as well as the normalized standard deviation $\sigma$. The former can be expressed as

$$
<\nu>=\sum_{\nu \geq 1} \nu n_{\nu} .
$$


The latter is

$$
\sigma^{2}=\frac{<\nu^{2}>-<\nu>^{2}}{<\nu>^{2}}
$$

where the average size squired $\left\langle\nu^{2}\right\rangle$ is

$$
<\nu^{2}>=\sum_{\nu \geq 1} \nu^{2} n_{\nu}
$$

\section{A. The free energy density functional}

The dimensionless Helmholtz free energy for cytoskeleton filaments $f$ can be written as a functional of density distributions as follow

$$
f=\beta F / N=f_{i g}+f_{i n t}+\sum_{\nu \geq 1} \int \beta \mu_{\nu}^{(0)} \rho_{\nu}(\omega) d \omega
$$

where $F$ is the free energy, $\beta=1 / k_{B} T$ the inverse temperature, $f_{i g}$ is the dimensionless ideal gas free energy, the second term $f_{\text {int }}$ is the dimensionless energy due to the inter-filament interactions, and $\mu_{\nu}^{(0)}$ is the standard chemical potential of $\nu$-sized filament.

Below we derivate the expressions for $f_{i g}$ and $f_{i n t}$ as functionals of the function densities $n_{\nu}$ and $\eta_{\nu}(\omega)$.

\section{Ideal-gas free energy $f_{i g}$}

The ideal-gas free energy per volume of the system $V$ can be written in the following form

$$
\frac{\beta F_{i g}}{V}=\sum_{\nu \geq 1} \int \rho_{\nu}(\omega)\left(\ln \left[4 \pi \rho_{\nu}(\omega) \Lambda_{\nu}^{3}\right]-1\right) d \omega
$$

where $\Lambda_{\nu}=\frac{h}{\sqrt{2 \pi m_{\nu} k_{B} T}}$ is a thermal deBroglie wavelength of $\nu$-sized filament, $h$ is Planck constant, and $m_{\nu}=\nu m_{1}$ the filament mass.

The substitution of Eq.(1) into Eq.(8) yields

$$
f_{i g}=\left(\ln \rho \Lambda_{1}^{3}-1\right) \sum_{\nu \geq 1} n_{\nu} \int \eta_{\nu}(\omega) d \omega+\sum_{\nu \geq 1} n_{\nu} \ln n_{\nu} \int \eta_{\nu}(\omega) d \omega+\sum_{\nu \geq 1} n_{\nu} s_{\nu}^{(o r)}+\int \rho_{\nu}(\omega) \ln \left(\nu^{-\frac{1}{3}}\right) d \omega(g
$$


where $f_{i g}=\beta F_{i g} / N$ is an ideal-gas free energy per filament $(N=\rho V), \Lambda_{1}=\frac{h}{\sqrt{2 \pi m_{1} k_{B} T}}$ a thermal deBroglie wavelength of a monomer, and $s_{\nu}^{(o r)}$ the orientational entropy (with sign minus)

$$
s_{\nu}^{(o r)}=\int \eta_{\nu}(\omega) \ln \left[4 \pi \eta_{\nu}(\omega)\right] d \omega
$$

\section{Interaction free energy $f_{\text {int }}$}

We write the interaction free energy per volume $V$ in a mean-field fashion, namely

$$
\frac{\beta F_{\text {int }}}{V}=\frac{1}{2} \sum_{\nu_{1} \geq 1} \sum_{\nu_{2} \geq 1} \int \rho_{\nu_{1}}\left(\omega_{1}\right) \rho_{\nu_{2}}\left(\omega_{2}\right) B_{\nu_{1} \nu_{2}}\left(\omega_{1}, \omega_{2}\right) d \omega_{1} d \omega_{2},
$$

In Eq.(11) $B_{\nu_{1} \nu_{2}}\left(\omega_{1}, \omega_{2}\right)$ is the cluster integral

$$
B\left(\omega_{1}, \omega_{2}\right)=\int d \vec{r}_{12}\left[1-e^{-\beta w_{12}\left(r_{12}, \omega_{1}, \omega_{2}\right)}\right]
$$

where $w_{12}$ is a potential of interaction between two filaments, and $r_{12}$ the closest distance between two filaments.

The expression (11) is a good approximation for the interaction free energy of monodisperse rigid charged rods $>$ Moreover, this expression becomes exact in the limit of infinitely long rods, i.e. for $L / D \rightarrow \infty$.

The substitution of Eq.(1) into Eq.(11) yields the following expression for $F_{\text {int }}$ as a functional of densities $n_{\nu}$ and $\eta_{\nu}(\theta)$

$$
\frac{\beta F_{i n t}}{V}=\frac{\rho^{2}}{2} \sum_{\nu_{1} \geq 1} \sum_{\nu_{2} \geq 1} n_{\nu_{1}} n_{\nu_{2}} \int \eta_{\nu_{1}}\left(\omega_{1}\right) \eta_{\nu_{2}}\left(\omega_{2}\right) B_{\nu_{1} \nu_{2}}\left(\omega_{1}, \omega_{2}\right) d \omega_{1} d \omega_{2}
$$

To calculate the cluster integral $B_{\nu_{1} \nu_{2}}\left(\omega_{1}, \omega_{2}\right)$ given in Eq.(12) in obvious form, we model the interaction potential between two charged rod filaments $w_{12}\left(r_{12}, \omega_{1}, \omega_{2}\right)$ by

$$
\beta w_{12}\left(r_{12}, \omega_{1}, \omega_{2}\right)= \begin{cases}+\infty, & r_{12}<D \\ \Gamma_{\perp}\left(r_{12}, \gamma_{12}\right), & D \leq r_{12}\end{cases}
$$


where $D$ is the rod diameter, and $\gamma_{12}$ the angle between them. The function $\Gamma\left(r_{12}, \gamma_{12}\right)$ represents the repulsive electrostatic inter-filament interaction potential [7]

$$
\Gamma_{\perp}\left(r_{12}, \gamma_{12}\right)=\frac{\Gamma_{\perp}}{\sin \gamma_{12}} e^{-k\left(r_{12}-D\right)},
$$

with coefficient $\Gamma_{\perp}$ is given by

$$
\Gamma_{\perp}=\frac{2 \pi \lambda^{2} \beta}{\varepsilon k_{B}} \frac{e^{-k_{B} D}}{\left[\frac{k_{B} D}{2} K_{1}\left(\frac{k_{B} D}{2}\right)\right]^{2}},
$$

and $k_{D}=\left(4 \pi \beta e^{2} / \varepsilon \sum_{i=1}^{2} \xi_{i} \rho_{i}\right)^{\frac{1}{2}}$ stands for the inverse Debye length, $K_{1}$ the modified Bessel function of 2 nd kind of 1 st order, $\lambda$ the linear charge density of a filament, $\varepsilon$ the dielectric permittivity of water solvent, $e$ the charge of electron, and $x i_{i}$ and $\rho_{i}$ the ion valency and concentration in solution.

Substitution of Eqs.(14) and (15) into Eq.(12) results in the following expression for the cluster integral $B_{\nu_{1}, \nu_{2}}\left(\omega_{1}, \omega_{2}\right)$

$$
B_{\nu_{1}, \nu_{2}}\left(\omega_{1}, \omega_{2}\right)=2 D_{e f f} l_{m}^{2} \nu_{1} \nu_{2} B^{*}\left(\sin \gamma_{12}\right)
$$

where the effective diameter $D_{\text {eff }}$ and the function $B^{*}\left(\sin \gamma_{12}\right)$ are given by Eqs.(A12) and (A14), respectively. The details on the calculations to get Eq.(17) are presented in Appendix A.

Furthermore, the substitution of Eq.(17) into Eq.(13) provides the following form for interaction free energy

$$
f_{\text {int }}=\rho \frac{\pi}{4} D_{\text {eff }} l_{m}^{2} \sum_{\nu_{1} \geq 1} \sum_{\nu_{2} \geq 1} n_{\nu_{1}} n_{\nu_{2}} \nu_{1} \nu_{2} h_{\nu_{1} \nu_{2}} .
$$

where $f_{\text {int }}=\beta F_{\text {int }} / N$ is the interaction free energy per filament and the function $h_{\nu_{1} \nu_{2}}$ is given by

$$
h_{\nu_{1} \nu_{2}}=\frac{4}{\pi} \int \eta_{\nu_{1}}\left(\omega_{1}\right) \eta_{\nu_{2}}\left(\omega_{2}\right) B^{*}\left(\sin \gamma_{12}\right) d \omega_{1} d \omega_{2} .
$$

Finally, the Lagrange functional $f^{\prime}$ is related to free energy functional $f$ in Eq.(7) by

$$
f^{\prime}=f+A\left(\sum_{\nu \geq 1} n_{\nu}-1\right)+B\left(\int \eta_{\nu}(\omega) d \omega-1\right)
$$


where the Lagrange multipliers $A$ and $B$ are due to the normalization conditions in Eqs.(2) and (3).

\section{B. The equilibrium size distribution function $n_{\nu}$}

For a fixed angular distribution function $\eta_{\nu}(\theta)$, the size distribution function $n_{\nu}$ is obtained by using the variational principle

$$
\frac{\partial f^{\prime}}{\partial n_{\nu}}=0
$$

The Ihs of Eq.(21) can be rewritten using of the chain rule in the following form

$$
\frac{\partial f^{\prime}}{\partial n_{\nu}}=\sum_{\lambda \geq 1} \mu_{\lambda}^{\prime}\left(\frac{\partial n_{\lambda}}{\partial n_{\nu}}\right)
$$

where $\mu_{\lambda}^{\prime}$ is

$$
\mu_{\lambda}^{\prime}=\frac{\partial f^{\prime}}{\partial n_{\lambda}}
$$

There is an additional connection between functions $\left\{n_{\nu}\right\}$ coming from Eq.(4) Differentiation on both sides of Eq.(4) gives us

$$
d n_{1}+\sum_{\nu \geq 2} \nu d n_{\nu}=0
$$

Thus, we can write Eq.(22) as follow

$$
\frac{\partial f^{\prime}}{\partial n_{\nu}}=\mu_{1}^{\prime} \frac{\partial n_{1}}{\partial n_{\nu}}+\sum_{\lambda \geq 2} \mu_{\lambda}^{\prime}\left(\frac{\partial n_{\lambda}}{\partial n_{\nu}}\right)
$$

The substitution of $d n_{1}$ from Eq.(24) into the rhs of Eq.(25) gives us

$$
\frac{\partial f^{\prime}}{\partial n_{\nu}}=-\mu_{1}^{\prime} \nu+\mu_{\nu}^{\prime}
$$


As a result, we have from Eqs.(21) and (26)

$$
\mu_{\nu}^{\prime}=\mu_{1}^{\prime} \nu
$$

It is worth noting that the set of $\nu$-sized filaments can be considered as a separated 'pseudophase'. Additionally, all the 'pseudo-phases' with all possible sizes $\nu$ are in equilibrium with each other if the equilibrium condition given by Eq.(27) is executed.

We substitute the free energy functional $f^{\prime}$ expression (20) into Eq.(23) and use Eqs.(7),(9),(18) to calculate the chemical potential $\mu_{\nu}^{\prime}$

$$
\beta \mu_{\nu}^{\prime}=\left(\ln \rho \Lambda_{1}^{3}+\ln n_{\nu}\right) \int \eta_{\nu}(\omega) d \omega+s_{\nu}^{(o r)}+\frac{\pi}{2} D_{e f f} l_{m}^{2} \rho \sum_{\nu^{\prime} \geq 1} n_{\nu^{\prime}} \nu \nu^{\prime} h_{\nu \nu^{\prime}}+\beta \mu_{\nu}^{(0)} \int \eta_{\nu}(\omega) d \omega+\mathcal{A}
$$

In Eq.(28), we use the rescaled standard chemical potential notation, i.e. $\beta \mu_{\nu}^{(0)} \rightarrow \beta \mu_{\nu}^{(0)}+$ $\ln \left(\nu^{-\frac{1}{3}}\right)$. Substitution of Eq.(3) into Eq.(28) gives us

$$
\beta \mu_{\nu}^{\prime}=\ln n_{\nu}-\ln C+\frac{\pi}{2} D l_{m}^{2} \rho \nu \sum_{\nu^{\prime} \geq 1} n_{\nu^{\prime}} \nu^{\prime} h_{\nu \nu^{\prime}}+\beta \mu_{\nu}^{(0)}
$$

where the constant $C$ is a constant independent of density distribution $n_{\nu}$

$$
-\ln C=\ln \rho \Lambda_{1}^{3}+s_{\nu}^{(o r)}+A
$$

To find the length distribution function $n_{\nu}$ we substitute the expression for the function $\mu_{\nu}^{\prime}(29)$ into the condition of equilibrium (27) getting

$$
\ln n_{\nu}-\ln C+\frac{\pi}{2} D l_{m}^{2} \rho \nu \sum_{\nu^{\prime} \geq 1} n_{\nu^{\prime}} \nu^{\prime} h_{\nu \nu^{\prime}}+\beta \mu_{\nu}^{(0)}=\nu\left(\ln n_{1}-\ln C+\frac{\pi}{2} D l_{m}^{2} \rho \sum_{\nu^{\prime} \geq 1} n_{\nu^{\prime}} \nu^{\prime} h_{1 \nu^{\prime}}+\beta \mu_{1}^{(0)}\right)
$$

which can be rewritten as follow

$$
\ln \frac{n_{\nu}}{C}=\nu \ln \frac{n_{1}}{C}-\frac{\pi}{2} D l_{m}^{2} \rho \nu \sum_{\nu^{\prime} \geq 1} n_{\nu^{\prime}} \nu^{\prime}\left(h_{\nu \nu^{\prime}}-h_{1 \nu^{\prime}}\right)-\beta \Delta \mu_{\nu}^{(0)}
$$


where $\Delta \mu_{\nu}^{(0)}$ denotes the standard chemical potential difference between $\nu$ actin units aggregated in a $\nu$-mer filament $\mu_{\nu}^{(0)}$, and the ones in singly dispersed phase $\nu \mu_{1}^{(0)}$, i.e.

$$
\Delta \mu_{\nu}^{(0)}=\mu_{\nu}^{(0)}-\nu \mu_{1}^{(0)}
$$

For $\nu=1$ we have

$$
\Delta \mu_{1}^{(0)}=0
$$

Finally, we introduce the parameter $y \equiv-\ln \left(\frac{n_{1}}{C}\right)$ and use it into Eq.(32)to have

$$
n_{\nu}=C e^{-\nu y} e^{-\beta \Delta \mu_{\nu}^{(0)}} e^{-\frac{\pi}{2} D l_{m}^{2} \rho \nu \sum_{\nu^{\prime} \geq 1} n_{\nu^{\prime}} \nu^{\prime}\left(h_{\nu \nu^{\prime}}-h_{1 \nu^{\prime}}\right)} .
$$

\section{The equilibrium angular distribution function $\eta_{\nu}(\omega)$}

Similarly, for a fixed distribution function $n_{\nu}$, the angular distribution function $\eta_{\nu}(\omega)$ is obtained by using the variational principle

$$
\frac{\partial f^{\prime}}{\partial \eta_{\nu}(\omega)}=0
$$

The substitution of the Lagrange functional $f^{\prime}$ expression (20) into the condition in Eq.(36) and the use of Eqs.(7),(9),(10),(18),(19)yield

$$
\begin{array}{r}
\ln \rho \Lambda^{3}-1+\sum_{\nu \geq 1} n_{\nu} \ln n_{\nu}+\sum_{\nu \geq 1} n_{\nu} \ln \eta_{\nu}\left(\omega_{1}\right)+\sum_{\nu \geq 1} n_{\nu} \\
+2 \rho D_{e f f} l_{m}^{2} \sum_{\nu_{1} \geq 1} \sum_{\nu_{2} \geq 1} n_{\nu_{1}} n_{\nu_{2}} \nu_{1} \nu_{2} \int \eta_{\nu_{2}}\left(\omega_{2}\right) \sin \gamma_{12} d \omega_{2}+\sum_{\nu \geq 1} \beta \mu_{\nu}^{(0)} n_{\nu}+B=0 .
\end{array}
$$

The use of the normalization condition (2) into the fourth term in Ihs of Eq.(37) lead to

$$
\sum_{\nu \geq 1} n_{\nu} \ln \eta_{\nu}\left(\omega_{1}\right)-\ln E+2 \rho D_{e f f} l_{m}^{2} \sum_{\nu_{1} \geq 1} \sum_{\nu_{2} \geq 1} n_{\nu_{1}} n_{\nu_{2}} \nu_{1} \nu_{2} \int \eta_{\nu_{2}}\left(\omega_{2}\right) \sin \gamma_{12} d \omega_{2}=0
$$


where $E$ is a constant independent on the angular density distribution $\eta_{\nu}(\omega)$,

$$
-\ln E=\ln \rho \Lambda^{3}+\sum_{\nu \geq 1} n_{\nu} \ln n_{\nu}+\sum_{\nu \geq 1} \beta \mu_{\nu}^{(0)} n_{\nu}+B .
$$

We substitute the expression for $\langle\nu\rangle$ (4) into Eq.(38) to have the following equation for $\eta_{\nu}(\omega)$

$$
\sum_{\nu \geq 1} n_{\nu} \eta_{\nu}\left(\omega_{1}\right)=E e^{-2 D l_{m}^{2} \rho<\nu>\sum_{\nu \geq 1} n_{\nu} \int \eta_{\nu}\left(\omega_{2}\right) \sin \gamma_{12} d \omega_{2}} .
$$

To find the normalization coefficient $E$ in r.h.s. of Eq.(40) we integrate both sides of Eq.(40) with respect to $\omega_{1}$ and use in the Ihs the normalization conditions (2) and (3)Finally, replacing the expression for $E$ into Eq.(40) we have the following relationship to find $\eta_{\nu}(\omega)$

$$
\sum_{\nu \geq 1} n_{\nu} \eta_{\nu}\left(\omega_{1}\right)=\frac{e^{-2 D_{e f f} l_{m}^{2} \rho<\nu>\sum_{\nu \geq 1} n_{\nu} \int \eta_{\nu}\left(\omega_{2}\right) \sin \gamma_{12} d \omega_{2}}}{\int d \omega_{1} e^{-2 D_{e f f} l_{m}^{2} \rho<\nu>\sum_{\nu \geq 1} n_{\nu} \nu \int \eta_{\nu}\left(\omega_{2}\right) \sin \gamma_{12} d \omega_{2}}} .
$$

It is worth noting that the angular distribution function $\eta_{\nu}(\omega)$ depends only on polar angle $\theta$ due to the symmetry of the system. This is accounted from the chosen nematic director, i.e. $\eta_{\nu}(\omega) \equiv \eta_{\nu}(\theta)$.

To find the size and angular distribution functions $n_{\nu}$ and $\eta_{\nu}(\theta)$, we numerically solve the expressions (35), (41), and (19) using the method of successive iterations described in appendix $\mathrm{H}$.

\section{Free energy in nematic phase}

In the nematic phase, the dimensionless free energy density $f$ has to be expressed as a functional of the Gaussian parameter $\alpha$ given by Eq. $(\mathrm{H} 13)$. To this end we substitute the distribution functions $n_{\nu}(\mathrm{H} 10)$ and $\eta_{\nu}(\theta)(\mathrm{H} 12)$, as well as the eq. $(\mathrm{H} 13)$ into the expressions(7),(9),(10),(18),(19).

\section{Ideal-gas free energy}

The substitution of the normalization conditions (2) and (3) into Eq.(9) gives us 


$$
f_{i g}=\ln \rho \Lambda^{3}-1+f_{m i x}+f_{\text {or }},
$$

where $f_{m i x}$ is the mixing free energy

$$
f_{\text {mix }}=\sum_{\nu \geq 1} n_{\nu} \ln n_{\nu}
$$

and $f_{\text {or }}$ the orientational free energy

$$
f_{o r}=\sum_{\nu \geq 1} n_{\nu} \int \eta_{\nu}(\theta) \ln \left[4 \pi \eta_{\nu}(\theta)\right] d \omega .
$$

We note that the mixing free energy $f_{m i x}$ is not well defined in the monodisperse limit, i.e. for $z \rightarrow \infty$. It should be zero. However, the density distribution $n_{\nu} \rightarrow \delta(\nu-\langle\nu\rangle)$, and according to Eq.(43), the mixing free energy diverges. To overcome this problem, we extract the density $\rho$ independent term $f_{m i x}(z \rightarrow \infty)$ in Eq.(C11) from the rhs of Eq.(43). In this case, we have

$$
f_{\text {mix }}=\sum_{\nu \geq 1} n_{\nu} \ln n_{\nu}+\ln [\sqrt{2 \pi e}<\nu>\sigma] .
$$

As a result, the new expression for the mixing free energy $f_{m i x}(45)$ recovers the correct value in the monodisperse limit, i.e. $f_{m i x} \rightarrow 0$ for $\sigma \rightarrow 0(z \rightarrow \infty)$. More details are provided in Appendix C.

To find the orientational free energy $f_{\text {or }}$ as a function of Gaussian parameter $\alpha$ given by Eq. $(\mathrm{H} 13)$ in obvious form we substitute the expressions for $\eta_{\nu}(\theta)(\mathrm{H} 12)$ and (H13) into Eq.(44). We have

$$
f_{\text {or }}=\ln \alpha-1-\frac{8}{9} \sigma^{2}
$$

The details of the calculation of Eq.(46) can be found in Appendix D. In a monodisperse limit when $\sigma \rightarrow 0$ the correct limit is obtained, i.e. $f_{\text {or }} \rightarrow \ln \alpha-1$ [20].

We note that the formula for $f_{\text {or }}$ in Eq.(44) is valid for rigid polyelectrolyte, i.e. for filaments with $L<<P$, where $P$ is the persistence length. For long filaments with $L>>P$ (semiflexible limit) the orientational free energy can be written in the following form 


$$
f_{\text {or }}=\sum_{\nu \geq 1} n_{\nu} \frac{L}{8 P} \int \frac{1}{\eta_{\nu}(\theta)}\left(\frac{\partial \eta_{\nu}(\theta)}{\partial \theta}\right)^{2} d \omega,
$$

The substitution of the expressions for $\eta_{\nu}(\theta)(\mathrm{H} 12)$ and (H13) into Eq.(47) gives us Eq.(E4), i.e.

$$
f_{\text {or }}=\frac{N_{p} \alpha}{4}
$$

where we define

$$
N_{p}=\frac{<L>}{P}\left(1+\frac{4}{3} \sigma^{2}\right) .
$$

and $\langle L\rangle=l_{m}\langle\nu\rangle$ (for details of these calculations see Appendix $\mathrm{E}$ ). In a monodisperse limit, $\sigma \rightarrow 0$, the correct limit is obtained, i.e. $f_{\text {or }} \rightarrow \frac{L}{P} \frac{\alpha}{4}[11]$.

To combine these two asymptotic cases for $f_{o r}$, we use the following interpolating formula

$$
f_{\text {or }}=\ln \alpha-\frac{8}{9} \sigma^{2}+N_{p} \frac{(\alpha-1)}{6}+\frac{5}{12} \ln \left[\cosh \left(N_{p} \frac{(\alpha-1)}{5}\right)\right]-\frac{19}{12} \ln 2 .
$$

The details on the calculation to get Eq.(50) are provided in Appendix E1.

\section{Free energy of interaction of filaments}

To find the interaction free energy $f_{\text {int }}$ as a function of the parameter $\alpha$ we substitute the expressions for $n_{\nu}(\mathrm{H} 10)$ and $\eta_{\nu}(\theta)(\mathrm{H} 12)$, as well as eq.(H13) into Eqs.(18) and (19). As a result we have

$$
f_{\text {int }}=\rho \frac{\pi}{4} D_{e f f} l_{m}^{2}<\nu>^{2} h_{z}
$$

where

$$
h_{z}=32 \int_{0}^{\pi / 2} \sin \theta_{1} d \theta_{1} \int_{0}^{\pi / 2} \sin \theta_{2} d \theta_{2} g_{z}\left(\theta_{1}\right) g_{z}\left(\theta_{2}\right) K\left(\theta_{1}, \theta_{2}\right)
$$

and 


$$
\begin{gathered}
g_{z}(\theta)=\frac{\alpha}{12 \pi} e^{\frac{\alpha}{6} \theta^{2}}\left(\frac{4 \frac{(z+2)}{(z+1)}}{\left(1+\frac{2 \alpha \theta^{2}}{3(z+1)}\right) z+3}-\frac{1}{\left(1+\frac{2 \alpha \theta^{2}}{3(z+1)}\right) z+2}\right) \\
K\left(\theta_{1}, \theta_{2}\right)=\int_{0}^{2 \pi} B^{*}\left(\sin \gamma_{12}\right) d \varphi_{12}
\end{gathered}
$$

The details on these calculations can be found in Appendix F.

In particular, the expression for $g_{z}(\theta)$ in monodisperse limit $z \rightarrow \infty(53)$ goes to

$$
g_{z}(\theta) \rightarrow \eta(\theta) \quad(z \rightarrow \infty)
$$

where $\eta(\theta)$ is the monodisperse distribution given by Eq.(H4). Similarly, Eqs.(51), (52), (53), (54) recover the correct expression for the free energy of interaction of filaments $f_{\text {int }}$

$$
f_{\text {int }}=\rho \frac{\pi}{4} D_{e f f} L^{2} h
$$

with

$$
h=32 \int_{0}^{\pi / 2} \sin \theta_{1} d \theta_{1} \int_{0}^{\pi / 2} \sin \theta_{2} d \theta_{2} \eta\left(\theta_{1}\right) \eta\left(\theta_{2}\right) K\left(\theta_{1}, \theta_{2}\right) .
$$

\section{E. Free energy in isotropic phase}

In the isotropic phase, there is no preferential direction, thus, the orientational distribution function $\eta_{\nu}(\theta)$ is given by

$$
\eta_{\nu}(\theta)=\frac{1}{4 \pi}
$$

and the orientational free energy

$$
f_{\text {or }}=0 \text {. }
$$

Substitution of Eq.(58) into Eq.(19) yields the function $h \equiv h_{\nu_{1} \nu_{2}}$ 


$$
h=\frac{4}{\pi} \frac{1}{(4 \pi)^{2}} \int B^{*}\left(\sin \gamma_{12}\right) d \omega_{1} d \omega_{2}
$$

The function $h$ in obvious form is provided by Eq.(G8). Substitution of Eq.(60) into Eq.(18) generate the following expression for the free energy of interaction of filaments in isotropic phase

$$
f_{\text {int }}=\rho \frac{\pi}{4} D_{\text {eff }} l_{m}^{2}<\nu>^{2} h
$$

Eq.(61) can also be expressed in terms of the second virial coefficient $B_{2}$

$$
f_{\text {int }}=\rho B_{2}
$$

where

$$
B_{2}=\frac{\pi}{4} D_{e f f} l_{m}^{2}<\nu>^{2} h
$$

\section{F. Isotropic-nematic phase equilibrium}

Using Eqs.(7),(42) and (51), the dimensionless free energy $f$ In the nematic and isotropic phases can be written as follow

$$
f(c)=\ln c-1+f_{\text {or }}+c h,
$$

where $c$ represents the dimensionless filaments density

$$
c=\rho \frac{\pi}{4} D_{e f f} l_{m}^{2}<\nu>^{2}
$$

In the nematic phase, the values for $f_{\text {or }}$ and $h \equiv h_{z}$ are given by Eqs.(50) and (52), accordingly. In contrary, they are given by Eqs.(59) and (G8) in the isotropic phase, respectively. We note that the mixing free energy $f_{m i x}$ and the term with $\mu_{\nu}^{(0)}$ in rhs of Eq.(64)are similar for both isotropic and nematic phases and, thus, do not contribute to the phase coexistence properties. In fact, the values for the coexisting densities $c_{I}$ and $c_{N}$, are of our interest. 
In the nematic phase, the expression for the free energy $f(64)$ is a function of Gaussian parameter $\alpha$ and the filaments density $c$, i.e. $f \equiv f(c ; \alpha)$. For each value of $c$ we use Eq.(H5) to calculate the Gaussian parameter at equilibrium $\alpha_{\min }(c)$, such as the nematic free energy at equilibrium is defined as $f(c)=f\left(c, \alpha_{\min }(c)\right)$.

The coexisting densities in isotropic and nematic phases, $c_{I}$ and $c_{N}$, are defined from the phase equilibrium conditions

$$
\mu_{1, N}\left(c_{N}\right)=\mu_{1, I}\left(c_{I}\right), \quad P_{N}\left(c_{N}\right)=P_{I}\left(c_{I}\right)
$$

In Eq.(66) $\mu_{1}(c)$ and $P(c)$ are the dimensionless chemical potential of monomers and osmotic pressure in isotropic and nematic phases

$$
\mu_{1}(c)=\frac{1}{<v>}\left(f(c)+\frac{P(c)}{c}\right), \quad P(c)=c^{2} \frac{\partial f(c)}{\partial c}
$$

\section{RESULTS}

In the numerical calculations we chose the experimental values for the filaments diameter $D=80 \AA$, the monomer units length $l_{m}=27 \AA$, the actin molar weight $m_{A}=42 k D a$, the monovalent electrolyte concentration $c_{e}=0.1 M$, and the linear charge density on filaments $\lambda=-4 \frac{e}{n m}$. Additionally, $G$-actin is usually polymerized in in-vitro experiments in the presence of gelsolin, an actin-binding protein to be known to cap and sever actin filaments. Thus, the variation of concentration of actin $\rho_{A}$ in experimental works is often accompanied by the variation of concentration of gelsolin $\rho_{G}$ to keep the degree of polymerization $\langle\nu\rangle$ to be the same in the isotropic and nematic phases $[14,15]$, where

$$
<\nu>=\frac{\rho_{A}}{\rho_{G}}
$$

Therefore, the average size $\langle\nu\rangle$ in experiments is an input parameter, which is governed by the ratio of concentrations of actin and gelsolin.

In Figure1 we plotted the isotropic-nematic phase diagram in term of coexisting densities of actin $\rho_{A}$ for different average length of filaments $\langle\nu\rangle$ and for two values of normalized standard deviation $\sigma=0$ (monodisperse) and $\sigma=0.3$ (weakly polydisperse) of Schulz's distribution 
function. In the same figure, we plotted the experimental data extracted from Figures 4 and 5 in Ref.[15]. Except for the average size $\langle\nu\rangle=8000$ in the nematic phase, our theoretical results fit well the experimental data. As it is mentioned in Ref.[15], the discrepancy for large filaments average sizes may arise from some obstacles that affects the correct measurement of the coexisting nematic densities. Fig.1 also shows that both coexisting densities of the polydisperse phase diagram with $\sigma=0.3$ are larger than the corresponding ones of the monodisperse phase diagram with $\sigma=0$. This is in part due to the experimental condition fixing the same degree of polymerization $\langle\nu\rangle$ for both the isotropic and nematic phases.

In Figure 2 we depicted the Gaussian parameter $\alpha$ of the nematic phase at phase equilibrium for different values of the parameter $\langle\nu\rangle$. For a given value of $\langle\nu\rangle$, the Gaussian parameter $\alpha$ decreases with increasing the polydispersity parameter $\sigma$.

To visualize the Schulz's distribution function $n_{\nu}$ for different average sizes $\langle\nu\rangle$ in a weakly polydisperse system, we depicted in Fig.3 $n_{\nu}$ for the normalized standard deviation $\sigma=0.3$ $(z=10)$ as a function of the filament size $\nu$. Our results reveal asymmetric distribution functions $n_{\nu}$ with lower peaks and broaden distributions for larger values of the average size $\langle\nu\rangle$. Additionally, they are very different from the delta-function distributions $n_{\nu} \rightarrow \delta(\nu-<\nu>)$ defined for the monodisperse case $\sigma=0$.

We also analyzed the impact of the persistence length $P$ in the phase diagram of actin filaments. It is experimentally estimated between $7-18 \mu \mathrm{m}$ depending on the polymerization buffers and experimental techniques. In Fig.4 we display the isotropic-nematic phase diagram for two values of persistence length, namely $P=7 \mu m$ and $P=18 \mu m$. For a given value $\langle\nu\rangle$, It is seen that longer values of the persistence length $P$ decrease both the isotropic and nematic coexisting densities. Additionally, the results for $P=18 \mu m$ gives the best fit against the experimental data. For a given value $\langle\nu\rangle$, we can conclude from Fig. 2 that longer persistence lengths $P$ lead to larger Gaussian parameters $\alpha$ in the coexisting nematic phase.

To study the effect of the electrolyte solution on the phase diagram, we plotted in Fig.5 the dependence of the effective diameter $D_{\text {eff }}$ on the concentration of monovalent ions $c_{e}$. It is seen that $D_{\text {eff }}$ exponentially decays with increasing $c_{e}$. This result agrees with the formation of an electrical double layer accumulating many counterions around the filament surface. The lower the electrolyte concentration, the larger its thickness and consequently, the effective filament diameter. Furthermore, we show in Fig.6 the isotropic-nematic phase diagram for two values of electrolyte concentration $c_{e}=0.01 M$ and $c_{e}=0.1 M$. Our results show that an increase in 
electrolyte concentration leads to an increase in both coexisting isotropic and nematic densities. Clearly, the results for $c_{e}=0.1 M$ give the best fit against the experimental data. For a given value $\langle\nu\rangle$, we conclude from Fig. 2 that the increase in the concentration $c_{e}$ slightly increases the Gaussian parameter $\alpha$ in the coexisting nematic phase.

\section{DISCUSSION}

In the present study we have developed a theory to calculate an isotropic-nematic phase diagram of actin filaments in an electrolyte solution. The approach is based on an extension of Onsager's second order theory for monodisperse, charged rigid rods [28]. The key ingredients in this work are in a unique definition for the orientational free energy $f_{o r}$, the $n_{\nu}$ size and angular $\eta_{\nu}(\theta)$ distribution functions, which properly account for the polydispersity and semiflexibility of the actin filaments.

Unlike many studies on polydisperse rods where the size distribution function $n_{\nu}$ is an input parameter $[19,22]$, actin filaments self-aggregate to produce a size distribution function $n_{\nu}$ that satisfies the equilibrium condition given in Eq.(27). Our results reveal that $n_{\nu}$ has a form of Schulz's distribution function, which is in accordance with experimental work done on actin filaments $[14,15]$.

To model an angular distribution function in nematic phase $\eta_{\nu}(\theta)$, we have used the trial function introduced in Ref.[19], which depends on a single parameter $\alpha$. The latter is obtained by minimizing the free energy functional expression given by Eq. $(\mathrm{H} 5)$.

We have accounted for the semiflexibility of long filaments by the generalization of the formula for orientational free energy $f_{\text {or }}$ introduced in Ref.[20] for polydisperse systems.

Finally, we have calculated and plotted the isotropic-nematic phase diagrams for some values of normalized standard deviation of Schult's distribution $\sigma$, the persistence length of actin filaments $P$ and the concentration of monovalent ions in electrolyte solution $c_{e}$. We have compared the obtained results with the corresponding experimental data presented in Ref.[15]. We have found that the set of parameters $P=18 \mu \mathrm{m}$ and $c_{e}=0.1 M$ gives the best match against the experiment, whereas the choice of parameter $\sigma \leq 0.3$ does not affect significantly the phase diagram.

Currently, we are considering several extensions of the approach along the following lines. We used the iterative procedure to find the distribution functions $n_{\nu}$ and $\eta_{\nu}(\theta)$ for weakly polydisperse 
systems, i.e. when the normalized standard deviation $\sigma$ is small. To extend the approach for highly polydisperse systems, the solution should be obtained without using the weak polydispersity approximation which, in turn, may increase the computational burden dramatically.

We have introduced an approximation for the excess standard chemical potential $\Delta \mu_{\nu}^{(0)}$. This is a reasonable approximation leading to Schulz's distribution function observed in experiments on actin filaments [14]. Indeed, the use of the approaches proposed for the amphiphilic micellas [31-33] could provide a more accurate expression for $\Delta \mu_{\nu}^{(0)}$ in actin filament solution.

We have used Brenner-Parsegian formula [7] to approximate the electrostatic part of interfilament potential interaction model. This approximation accounts for water solvent in form of a dielectric continuum characterized by a constant dielectric permittivity. A more accurate result for the free energy and phase diagrams could be obtained using the DFT method introduced in Ref. [5] to describe the electrostatic interaction between filaments, where the solvent water molecules are modeled as molecular dipoles capturing the delicate peculiarities of the water solvent and dielectric permittivity profile near the surface of filaments.

In our method we have accounted only repulsive interaction between filaments, i.e the hard core plus electrostatic repulsions in Eq.(14). However, actin filaments may form bundles and networks due to the attraction forces produced by linker proteins or divalent ions between filaments. Thus, Future work requires the extension of our theory to account for both attractive and repulsive interfilament potential interactions. The attraction between filaments can be modeled, for example, using the following potential $[4,8]$

$$
\beta w_{a t t}\left(r_{12}, \omega_{1}, \omega_{2}\right)= \begin{cases}+\infty, & r_{12}<D, \\ -\frac{\epsilon}{\sin \gamma_{12}}, & D \leq r_{12}<D+\delta, \\ 0, & D+\delta \leq r_{12}\end{cases}
$$

where $\epsilon>0$ is a strength of attraction and $\delta$ the range of attraction. This extension will allow to analyze cytoskeleton conformation changes from alterations in the number density of actins/tubulin, number density and type of binding agents, and electrolyte concentration covering the full phase diagrams, including the bundling and networking phases.

Finally, the approach and their extensions could be modified to investigate the phase diagrams of other polyelectrolyte solutions including microtubules, intermediate filaments, DNAs, and 
tabacco virus.

\section{Acknowledgements}

This work was supported by the National Institutes of Health [grant number 1SC1GM127187].

[1] B.Albert, A.D.Johnson, et al, Molecular Biology of the Cell, 6th Ed., 2014, P.1465.

[2] A.Suzuki, M.Yamazaki, and T.Ito, Biochem. 35, 5238(1996).

[3] J.Tang, P.Janmey, J.Bio.Chem. 271, 8556(1996).

[4] I.Borukhov, R.Bruinsma,W.Gelbart, and A.Liu, PNAS, 102, 3673(2005).

[5] V.Warshavsky, and M.Marucho, PRE, 93, 042607(2016).

[6] L.Onsager, Ann NY Acad Sci, 51, 627(1949).

[7] S.Brenner and V.Parsegian, Biophys.J., 14, 327(1974).

[8] A.Grosberg, and A.Khokhlov, Adv.Polym.Sci., 41, P.53.

[9] A.Khokhlov and A.Semenov, Physica A, 108, 546(1981).

[10] A.Khokhlov and A.Semenov, Physica A, 112, 605(1982).

[11] G.Vroege and H.Lekkerkerker, Rep.Prog.Phys., 55, 1241(1992).

[12] A.Stroobants, H.N.W.Lekkerkerker, and Th.Odijk, Macromolecules, 19, 2232(1986).

[13] P.Janmey, J.Peetermans, K.Zaner, T.Stossel, and T.Tanaka, J.Bio.Chem., 261, 8357(1986).

[14] J.Drogemeier, H.Hinsen, and W.Eimer, Macromol, 27,87(1994).

[15] A.Suzuki, T.Maeda, and T.Ito, Biophys.J., 59, 25(1991).

[16] X.Qiu,K.Andresen,L.Kwok,J.Lamb,H.Y.Park, and L.Pollack, PRL, 99, 038104(2007).

[17] E.Yarmola, M.Zarudnaya, and Yu.Lazurkin, JBSD, 2, 981(1985).

[18] D.Stigter, Biopolymers, 16, 1435(1977).

[19] T.Sluckin, Liquid Cryst.,6, 111(1989).

[20] T.Odijk, Macromolecules, 19, 2313(1987).

[21] R.Hentschke, Macromolecules, 23, 1192(1990).

[22] A.Speranza and P.Sollich, JCP,117, 5421(2002).

[23] H.Wensink and G.Vroege, J.Chem.Phys.,119, 6868(2003).

[24] A.Speranza and P.Sollich, Phys.Rev.E, 67, 061702(2003). 
[25] E.Ruckenstein and R.Nagarajan, JPC, 85, 3010(1981)

[26] G. Oster, J Gen Physiol. 33(5), 445-473(1950).

[27] R.Hentschke, Macromol. 23, 1192(1990). 5

[28] L. Onsager, Ann. NY Acad. Sci., 51, 627(1949).

[29] D.Biron,E.Moses,I.Borukhov,S.A.Safran, Europhys.Lett., 73, 464(2006).

[30] B.Zimm, J.Chem.Phys., 16, 1099(1948).

[31] R.Nagarajan, E.Ruckensten, Langmuir, 7, 2934(1991).

[32] W.E.McMullen, W.M.Gelbart, A.Ben-Shaul, J.Chem.Phys., 82, 5616(1985).

[33] W.E.McMullen, W.M.Gelbart, A.Ben-Shaul, J.Phys.Chem., 88, 6649(1984).

[34] I. S. Gradshteyn, I. M. Ryzhik, A.Jeffrey, D. Zwillinger Table of Integrals, Series, and Products.

\section{Appendix A: Calculation of the cluster integrals $B\left(\omega_{1}, \omega_{2}\right)$}

The volume integration in Eq.(12) can be written as follow

$$
\int d \vec{r}_{12} \rightarrow \int_{0}^{L_{1}} d z \int_{0}^{L_{2} \sin \gamma_{12}} d y \int_{-\infty}^{+\infty} d x
$$

where the axis $x$ is chosen along the direction of the closest distance between rods, the axis $z$ along the direction of 1 st rod, and the axis $y$ in the perpendicular direction to $z-x$ plane. $L_{1}$, $L_{2}$ are the lengths of two rods, and $\gamma_{12}$ the angle between the axis of two rods.

The substitution of Eq.(A1) into Eq.(12) gives

$$
B\left(\omega_{1}, \omega_{2}\right)=\int_{0}^{L_{1}} d z \int_{0}^{L_{2} \sin \gamma_{12}} d y \int_{-\infty}^{+\infty} d x\left[1-e^{-\beta w_{12}\left(x, \omega_{1}, \omega_{2}\right)}\right] .
$$

The integration in Eq.(A2) in $y-z$ plane gives

$$
B\left(\omega_{1}, \omega_{2}\right)=L_{1} L_{2} \sin \gamma_{12} 2 \int_{0}^{+\infty} d x\left[1-e^{-\beta w_{12}\left(x, \omega_{1}, \omega_{2}\right)}\right] .
$$

The substitution of Eq.(14) into Eq.(A3) yields

$$
B\left(\omega_{1}, \omega_{2}\right)=2 L_{1} L_{2} \sin \gamma_{12}\left(D+\int_{D}^{+\infty} d x\left[1-e^{-\Gamma_{\perp}\left(x, \gamma_{12}\right)}\right]\right) .
$$

The substitution ofEq.(15) into the integral in the rhs of Eq.(A4) yields the following expression 


$$
J=\int_{D}^{+\infty} d x\left[1-e^{-C e^{-k x}}\right]
$$

where

$$
C=\frac{\Gamma_{\perp}}{\sin \gamma_{12}} e^{k D}
$$

Eq.(A5) is solved analytically to get

$$
J=\frac{1}{k}\left\{\ln \left(C e^{-k D}\right)+\gamma_{E}+E_{1}\left(C e^{-k D}\right)\right\},
$$

where $\gamma_{E}$ is Euler's constant

$$
\gamma_{E}=-\int_{0}^{+\infty} e^{-t} \ln t d t=0.5772 \ldots
$$

and $E_{1}(x)$ is an exponential integral

$$
E_{1}(x)=\int_{x}^{+\infty} \frac{e^{-t}}{t} d t
$$

Next, the substitution of Eqs.(A7),(A6) into Eq.(A4) gives

$$
B\left(\omega_{1}, \omega_{2}\right)=2 L_{1} L_{2} \sin \gamma_{12}\left(D+\frac{1}{k}\left\{\ln \left(\frac{\Gamma_{\perp}}{\sin \gamma_{12}}\right)+\gamma_{E}+E_{1}\left(\frac{\Gamma_{\perp}}{\sin \gamma_{12}}\right)\right\}\right)
$$

We introduce the effective diameter $D_{\text {eff }}$ to rewrite Eq.(A10) as

$$
B\left(\omega_{1}, \omega_{2}\right)=2 L_{1} L_{2} \sin \gamma_{12} D_{e f f}\left(1+\frac{1}{k D_{e f f}}\left\{-\ln \sin \gamma_{12}+E_{1}\left(\frac{\Gamma_{\perp}}{\sin \gamma_{12}}\right)\right\}\right),
$$

where

$$
D_{\text {eff }}=D+\frac{1}{k}\left\{\ln \left(\Gamma_{\perp}\right)+\gamma_{E}\right\}
$$

Finally, Eq.(A11) can be written as

$$
B\left(\omega_{1}, \omega_{2}\right)=2 D_{e f f} l_{m}^{2} \nu_{1} \nu_{2} B^{*}\left(\sin \gamma_{12}\right)
$$

where

$$
B^{*}\left(\sin \gamma_{12}\right)=\sin \gamma_{12}\left\{1-\frac{1}{k D_{e f f}} \log \left(\sin \gamma_{12}\right)+\frac{1}{k D_{e f f}} E_{1}\left(\frac{\Gamma_{\perp}}{\sin \gamma}\right)\right\}
$$




\section{Appendix B: Properties of Schulz's distribution function}

Here, we provide with some properties of Schulz's distribution function $n_{\nu}$ from Eq.(H10). The average size in $n$-th power $\left\langle\nu^{n}>\right.$ is

$$
<\nu^{n}>=\int_{0}^{\infty} d \nu \nu^{n} n_{\nu}
$$

The substitution of Eq.(H10) into Eq.(B1) gives

$$
<\nu^{n}>=\frac{\Gamma(z+n+1)}{\Gamma(z+1)} \frac{1}{y^{n}}
$$

where $\Gamma$ is Gamma-function

$$
\Gamma(z+1)=\int_{0}^{\infty} d x x^{z} e^{-x} .
$$

From Eq.(B2) we have the correct normalization condition of Schult's distribution function for $n=0$

$$
<\nu^{0}>=<1>=1
$$

For $n=1$ we have the average length $\langle\nu\rangle$

$$
<\nu=\frac{(z+1)}{y} .
$$

and for $n=2$ we have the average squared length $\left\langle\nu^{2}\right\rangle$

$$
<\nu^{2}>=\frac{(z+1)(z+2)}{y^{2}} .
$$

The substitution of Eqs.(B5) and (B6) into the definition of the normalized standard deviation $\sigma$ in Eq.(5) gives

$$
\sigma=\frac{1}{\sqrt{z+1}}
$$




\section{Appendix C: Asymptotic calculation for $f_{m i x}$}

In this Appendix we calculate the asymptotic behavior of the mixing entropy $f_{m i x}$ in monodisperse limit, i.e. for $z \rightarrow \infty$. To this end we substitute the distribution function $n_{\nu}$, given by Eq.(H10), into Eq.(43). We have

$$
\begin{gathered}
f_{\text {mix }}=\int d \nu n_{\nu} \ln \left(\frac{y^{z+1}}{\Gamma(z+1)} \nu^{z} e^{-y \nu}\right) \\
f_{\text {mix }}=\ln \left[\frac{y^{z+1}}{\Gamma(z+1)}\right] \int d \nu n_{\nu}+z \int d \nu n_{\nu} \ln \nu-y \int d \nu n_{\nu} \nu
\end{gathered}
$$

The substitution of $<1>=\int d \nu n_{\nu}$ from Eq.(B4) in the first term in rhs of Eq.(C2), $n_{\nu}$ from Eq. $(\mathrm{H} 10)$ in the second term in rhs of Eq.(C2), and $\langle\nu\rangle=\int d \nu \nu n_{\nu}$ from Eq.(B5) into the third term in rhs of Eq.(C2) yields

$$
f_{\text {mix }}=\ln \left[\frac{y^{z+1}}{\Gamma(z+1)}\right]+z \frac{y^{z+1}}{\Gamma(z+1)} J-(z+1)
$$

where

$$
J=\int d \nu \nu^{z} e^{-y \nu} \ln \nu
$$

We calculate $J$ from Eq.(C4) using the integral Table, expression number 4.352 from Ref.[34]. We have

$$
J=\frac{\Gamma(z+1)}{y^{z+1}}\left(H_{z}-\gamma_{E}-\ln y\right) .
$$

where $\gamma_{E}=0.5772$ is Euler's constant, $\Gamma(z+1)=z$ ! the gamma-function, and $H_{z}$ the $z$-th harmonic number

$$
H_{z}=1+\frac{1}{2}+\frac{1}{3}+\ldots+\frac{1}{z}
$$

The substitution ofEq.(C5) into the second term in rhs of Eq.(C3) gives 


$$
f_{\text {mix }}=\ln \left[\frac{y}{\Gamma(z+1) e^{z+1}}\right]+z\left(H_{z}-\gamma_{E}\right)
$$

We use the asymptotic formula for harmonic number

$$
H_{z} \rightarrow \ln z+\gamma_{E}+\frac{1}{2 z} \quad(z \rightarrow \infty)
$$

to calculate the free energy $f_{\text {mix }}$ in the monodisperse limit, i.e. for the case when $z \rightarrow \infty$.

We substitute Eq.(C8) into Eq.(C7) to have

$$
f_{\text {mix }} \rightarrow \ln \left[\frac{y z^{z}}{\Gamma(z+1) e^{z+\frac{1}{2}}}\right], \quad(z \rightarrow \infty)
$$

Using Stirling's formula $\Gamma(z+1) \rightarrow \sqrt{2 \pi z}(z / e)^{z}$ in rhs of Eq.(C9), we obtain

$$
f_{\text {mix }} \rightarrow \ln \left[\frac{y}{\sqrt{2 \pi z e}}\right] \quad(z \rightarrow \infty)
$$

Finally, we substitute Eqs.(B5) and (B7) into Eq.(C10) to get

$$
f_{\text {mix }} \rightarrow-\ln \left[\sqrt{\frac{2 \pi e z}{z+1}}<\nu>\sigma\right] \quad(z \rightarrow \infty) .
$$

\section{Appendix D: Expression for $f_{o r}$ in Eq.(46)}

Here, we explain how to calculate the orientational free energy $f_{\text {or }}$ given by Eq.(46). To this end we substitute Eq.(H12) into Eq.(44). As a result, we have

$$
f_{\text {or }}=\sum_{\nu \geq 1} n_{\nu} 2 \int_{0}^{\frac{\pi}{2}} \frac{\alpha_{\nu}}{4 \pi} e^{-\frac{\alpha_{\nu}}{2} \theta^{2}} \ln \left(4 \pi \frac{\alpha_{\nu}}{4 \pi} e^{-\frac{\alpha_{\nu}}{2} \theta^{2}}\right) 2 \pi \sin \theta d \theta
$$

The simplification of Eq.(D1) leads to

$$
f_{o r}=\sum_{\nu \geq 1} n_{\nu}\left(\ln \alpha_{\nu}-1\right)
$$


The substitution of, Eq.(H13) into rhs of Eq.(D2), the use of the normalization condition given by Eq.(2) and the expansion of the logarithm function up to second order in powers of the small parameter $\frac{\Delta \nu}{\langle\nu\rangle}<<1$ give

$$
f_{\text {or }}=\ln \alpha-1+\sum_{\nu \geq 1} n_{\nu}\left(\frac{4}{3} \frac{\Delta \nu}{<\nu>}-\frac{1}{2} \frac{16}{9} \frac{(\Delta \nu)^{2}}{\langle\nu\rangle^{2}}\right),
$$

where $\Delta \nu=\nu-\langle\nu\rangle$.

Using Eqs.(4),(5) and (6) in the rhs of Eq.(D3) finally we obtain

$$
f_{\text {or }}=\ln \alpha-1-\frac{8}{9} \sigma^{2}
$$

\section{Appendix E: Expression for $f_{o r}$ in Eq.(48)}

In this Appendix we describe how to transform the expression (47) for the orientational free energy $f_{o r}$ to obtain Eq.(48). To this end, the substitution of $\eta_{\nu}(\theta)$ in Eq.(H12) into Eq.(47) produces

$$
\begin{gathered}
f_{\text {or }}=\sum_{\nu \geq 1} n_{\nu} \frac{L}{8 P} 4 \pi \int_{0}^{\frac{\pi}{2}} \frac{\alpha_{\nu}^{3}}{4 \pi} \theta^{2} e^{-\frac{\alpha_{\nu}}{2} \theta^{2}} \sin \theta d \theta, \\
f_{\text {or }}=\sum_{\nu \geq 1} n_{\nu} \frac{L}{4 P} \alpha_{\nu} .
\end{gathered}
$$

We substitute the parameter $\alpha_{\nu}$ in Eq.(H13) into Eq.(E2), and we use the condition $L=l_{m} \nu$ to give

$$
f_{\text {or }}=\sum_{\nu \geq 1} n_{\nu} \frac{l_{m} \nu}{4 P} \alpha\left(1+\frac{4}{3} \frac{\Delta \nu}{<\nu>}\right) .
$$

Finally, using Eqs.(4),(5), and (6) in Eq.(E3) we obtain

$$
f_{\text {or }}=\frac{l_{m}<\nu>}{P} \frac{\alpha}{4}\left(1+\frac{4}{3} \sigma^{2}\right)
$$

\section{1. $f_{o r}$ in Eq.(50)}

The orientational free energy $f_{\text {or }}$ in the nematic phase for monodisperse systems can be expressed as 


$$
f_{\text {or }}= \begin{cases}\int \eta \ln \eta d \omega, & L \ll P \\ \frac{L}{8 P} \int \frac{1}{\eta}\left(\frac{\partial \eta}{\partial \theta}\right)^{2} d \omega, & L \gg P\end{cases}
$$

where $L$ is the filament length, and $P$ the persistence length. Khokhlov and Semenov introduced the correction terms to the rhs of Eq.(E5) to make this expression even more accurate

$$
f_{\text {or }}= \begin{cases}\int \eta \ln \eta d \omega+\frac{L}{12 P} \int \frac{1}{\eta}\left(\frac{\partial \eta}{\partial \theta}\right)^{2} d \omega, & L \ll P \\ \frac{L}{8 P} \int \frac{1}{\eta}\left(\frac{\partial \eta}{\partial \theta}\right)^{2} d \omega-2 \ln \left[\int \eta^{\frac{1}{2}} d \omega\right]+\ln [4 \pi] & L \gg P\end{cases}
$$

We generalize Eq.(E6) to the case of polydisperse system in the following form

$$
f_{o r}= \begin{cases}\sum_{\nu \geq 1} n_{\nu} \int \eta_{\nu} \ln \eta_{\nu} d \omega+\sum_{\nu \geq 1} n_{\nu} \frac{L}{12 P} \int \frac{1}{\eta_{\nu}}\left(\frac{\partial \eta_{\nu}}{\partial \theta}\right)^{2} d \omega, & <L>\ll P \\ \sum_{\nu \geq 1} n_{\nu} \frac{L}{8 P} \int \frac{1}{\eta_{\nu}}\left(\frac{\partial \eta_{\nu}}{\partial \theta}\right)^{2} d \omega-2 \sum_{\nu \geq 1} n_{\nu} \ln \left[\int \eta_{\nu}^{\frac{1}{2}} d \omega\right]+\ln [4 \pi], & <L>\gg P\end{cases}
$$

where $\langle L\rangle=l_{m}\langle\nu\rangle$. The substitution of the distribution function $\eta_{\nu}(\theta)$ from Eq. $(\mathrm{H} 12)$ into Eq.(E7) and the use of Eqs.(46) and (48) lead to

$$
f_{\text {or }}= \begin{cases}\ln \alpha-1-\frac{8}{9} \sigma^{2}+N_{p} \frac{\alpha}{6}, & <L>\ll P \\ N_{p} \frac{\alpha}{4}+\ln \alpha-\frac{8}{9} \sigma^{2}-2 \ln 2, & <L>\gg P\end{cases}
$$

where $N_{p}$ is given by Eq.(49).

We combines the two asymptotic values in Eq.(E8) obtaining

$$
f_{\text {or }}=\ln \alpha-\frac{8}{9} \sigma^{2}+N_{p} \frac{\alpha}{6}+\frac{5}{12} \ln \left[\cosh \left(N_{p} \frac{\alpha}{5}\right)\right]-\frac{19}{12} \ln 2 .
$$

Eq.(E9) reproduces Eq.(E8) for two limiting cases $N_{p} \rightarrow 0(<L>\ll P)$ and $N_{p} \rightarrow \infty$ $(<L>\gg P)$. Additionally, in monodisperse case, i.e. for $\sigma=0$, Eqs.(49), (E8) and (E9) reduce to those provided in Ref.[20]. It was also suggested to substitute $(\alpha-1) N_{p}$ instead $\alpha N_{p}$ in Ref.[20] to have a reliable results for small $\alpha$ even when $N_{p} \sim 1$. 


\section{Appendix F: Expression for $f_{i n t}$ in Eqs. (51)}

Here we explain how to obtain the interaction free energy $f_{\text {int }}$ in Eqs. (51), (52), (53), (54). To this end, we use Eqs.(18),(19) to have

$$
f_{\text {int }}=\rho \frac{\pi}{4} D_{e f f} l_{m}^{2} \frac{4}{\pi} \int \sum_{\nu_{1} \geq 1} n_{\nu_{1}} \nu_{1} \eta_{\nu_{1}}\left(\theta_{1}\right) \sum_{\nu_{2} \geq 1} n_{\nu_{2}} \nu_{2} \eta_{\nu_{2}}\left(\theta_{2}\right) B^{*}\left(\sin \gamma_{12}\right) d \omega_{1} d \omega_{2},
$$

Eq.(F1) can be expressed as

$$
f_{i n t}=\rho \frac{\pi}{4} D_{e f f} l_{m}^{2}<\nu>^{2} \frac{4}{\pi} \int g_{z}\left(\theta_{1}\right) g_{z}\left(\theta_{2}\right) B^{*}\left(\sin \gamma_{12}\right) d \omega_{1} d \omega_{2}
$$

where

$$
g_{z}(\theta)=\frac{1}{<\nu>} \sum_{\nu \geq 1} n_{\nu} \nu \eta_{\nu}(\theta)
$$

Additionally, we substitute the distribution functions $n_{\nu}$ from Eq. $(\mathrm{H} 10)$ and $\eta_{\nu}(\theta)$ from Eqs.(H12) and (H13) into Eq.(F3). It results

$$
g_{z}(\theta)=\frac{1}{<\nu>} \sum_{\nu \geq 1} \frac{y^{z+1}}{\Gamma(z+1)} \nu^{z} e^{-y \nu} \nu \frac{\alpha}{4 \pi<\nu>} \frac{1}{3}\left(\frac{4 \nu}{<\nu>}-1\right) e^{-\frac{\alpha}{2} \frac{1}{3}\left(\frac{4 \nu}{<\nu>}-1\right) \theta^{2}} .
$$

The summation in Eq.(F4) can be written in the form of integration as follow

$$
g_{z}(\theta)=\frac{y^{z+1}}{\Gamma(z+1)} \frac{1}{4 \pi} \frac{\alpha}{<\nu>^{2}} \frac{1}{3} e^{\frac{\alpha}{6} \theta^{2}} \int_{0}^{\infty} d \nu\left(4 \nu^{z+2}-\nu^{z+1}<\nu>\right) e^{-\left(y+\frac{\alpha}{2} \frac{4}{3<\nu>} \theta^{2}\right) \nu}
$$

Using the definition of the Gamma-function, Eq.(B3), in Eq.(F5) we have

$$
g_{z}(\theta)=\frac{\alpha}{12 \pi} \frac{y^{z+1}}{<\nu>^{2}} e^{\frac{\alpha}{6} \theta^{2}}\left(\frac{\Gamma(z+3)}{\Gamma(z+1)} \frac{4}{\left(y+\frac{2 \alpha \theta^{2}}{3<\nu>}\right)^{z+3}}-\frac{\Gamma(z+2)}{\Gamma(z+1)} \frac{<\nu>}{\left(y+\frac{2 \alpha \theta^{2}}{3<\nu>}\right)^{z+2}}\right) .
$$

which can be rewritten as

$$
g_{z}(\theta)=\frac{\alpha}{12 \pi} \frac{e^{\frac{\alpha}{6} \theta^{2}}}{<\nu>^{2}}\left(\frac{4(z+1)(z+2)}{y^{2}\left(1+\frac{2 \alpha \theta^{2}}{3<\nu>y}\right)^{z+3}}-\frac{(z+1)<\nu>}{y\left(1+\frac{2 \alpha \theta^{2}}{3<\nu>y}\right)^{z+2}}\right)
$$


The substitution of the function $y$ in Eq.(B5) into Eq.(F7) gives

$$
g_{z}(\theta)=\frac{\alpha}{12 \pi} e^{\frac{\alpha}{6} \theta^{2}}\left(\frac{4 \frac{(z+2)}{(z+1)}}{\left(1+\frac{2 \alpha \theta^{2}}{3(z+1)}\right) z+3}-\frac{1}{\left(1+\frac{2 \alpha \theta^{2}}{3(z+1)}\right) z+2}\right) .
$$

Finally, we transform the angular integrations in Eq.(F2). To this end we use the following formula

$$
\int d \omega_{1} d \omega_{2} \ldots=8 \pi \int_{0}^{\pi / 2} \sin \theta_{1} d \theta_{1} \int_{0}^{\pi / 2} \sin \theta_{2} d \theta_{2} \int_{0}^{2 \pi} d \varphi_{12} \ldots
$$

Substitution Eq.(F9) into Eq.(F2) finally yields

$$
f_{i n t}=\rho \frac{\pi}{4} D l_{m}^{2}<\nu>^{2} 32 \int_{0}^{\pi / 2} \sin \theta_{1} d \theta_{1} \int_{0}^{\pi / 2} \sin \theta_{2} d \theta_{2} g_{z}\left(\theta_{1}\right) g_{z}\left(\theta_{2}\right) K\left(\theta_{1}, \theta_{2}\right)
$$

where

$$
K\left(\theta_{1}, \theta_{2}\right)=\int_{0}^{2 \pi} B^{*}\left(\sin \gamma_{12}\right) d \varphi_{12}
$$

\section{Appendix G: Expression for $h$ in Eq.(60)}

We start with the expression (60) for the function $h$. This function can be expressed in the following form

$$
h=\frac{2}{\pi} \int_{0}^{\pi} B^{*}\left(\gamma_{12}\right) \sin \gamma_{12} d \gamma_{12}
$$

Substitution of $B^{*}\left(\sin \gamma_{12}\right)$ from Eq.(A14) into Eq.(G1) gives us

$$
h=\frac{2}{\pi}\left(I_{1}-\frac{1}{k D_{\text {eff }}} I_{2}+\frac{\pi}{2} \frac{1}{k D_{\text {eff }}} I_{4}\right),
$$

where

$$
I_{1}=\int_{0}^{\pi} d \gamma_{12} \sin ^{2} \gamma_{12}
$$




$$
\begin{gathered}
I_{2}=\int_{0}^{\pi} d \gamma_{12} \sin ^{2} \gamma_{12} \ln \left(\sin \gamma_{12}\right) \\
I_{4}=\frac{2}{\pi} \int_{0}^{\pi} d \gamma_{12} \sin ^{2} \gamma_{12} E_{1}\left(\frac{\Gamma_{\perp}}{\sin \gamma_{12}}\right)
\end{gathered}
$$

The integrals $I_{1}$ and $I_{2}$ in Eqs.(G3) and (G4) can be calculated analytically (see integral table, number 4.384 .3 in Ref.[34])

$$
\begin{gathered}
I_{1}=\frac{\pi}{2} \\
I_{2}=\frac{\pi}{2}\left(-\log (2)+\frac{1}{2}\right)
\end{gathered}
$$

Finally, the substitution of Eqs.(G6) and (G7) into Eq.(G2) gives

$$
h=1+\frac{1}{k D_{e f f}}\left[\log (2)-\frac{1}{2}\right]+\frac{1}{k D_{e f f}} I_{4} .
$$

\section{Appendix H: Iteration method}

We chose the initial guess $n_{\nu}^{(1)}$ for the first iterative step and substitute this value in both sides of Eq.(41). The resulting equation is solved to calculate $\eta_{\nu}^{(1)}(\theta)$. During the second step, we substitute $n_{\nu}^{(1)}$ and $\eta_{\nu}^{(1)}(\theta)$ in rhs of Eq.(35) to obtain $n_{\nu}^{(2)}$ in Ihs of Eq.(35) and so on. We consider weakly polydisperse filaments, thus, we obtain a monodisperse distribution in the first iterative step. Higher-order iterative steps provide small corrections to that monodisperse distribution. It will be shown that the corrections are of the order of the normalized standard deviation $\sigma<<1$, which can be considered as a small parameter in the iterations.

\section{a. 1st iteration}

We choose the monodisperse distribution as a first iteration for $n_{\nu}$, i.e.

$$
n_{\nu}=\delta(\nu-<\nu>)
$$


where $\delta$ is a Dirac delta-function.

The substitution of Eq.(H1) into Eq.(41) generates the following equation to find $\eta(\theta)$

$$
\eta\left(\theta_{1}\right)=\frac{e^{-2 D_{e f f} l_{m}^{2} \rho<\nu>^{2} \int \eta\left(\theta_{2}\right) \sin \gamma_{12} d \omega_{2}}}{\int d \omega_{1} e^{-2 D_{e f f} l_{m}^{2} \rho<\nu>^{2} \int \eta\left(\theta_{2}\right) \sin \gamma_{12} d \omega_{2}}} .
$$

In this approximation all filaments have the same size $\nu \equiv\langle\nu\rangle$ and we use a notation for the angular distribution function $\eta(\theta) \equiv \eta_{<\nu>}(\theta)$. Eq. $(\mathrm{H} 2)$ can be solved numerically for the unknown function $\eta(\theta)$ using the iteration method.

More efficient but less accurate values for the angular distribution function $\eta(\theta)$ can also be obtained in some functional form. Onsager introduced the trial function

$$
\eta(\theta)=\frac{\alpha}{4 \pi} \frac{\cosh (\alpha \cos \theta)}{\sinh \alpha} .
$$

Another commonly used although slightly less accurate anzatz is the so-called Odijk's trial function

$$
\eta(\theta)=\frac{\alpha}{4 \pi} e^{-\frac{\alpha}{2} \theta^{2}}
$$

These trial functions have a single unknown parameter $\alpha$, which can be found by the minimization of the free energy functional $f$ with parameter $\alpha$. In other words, $\alpha_{\min }$ should be found to satisfy the condition

$$
f\left(\alpha_{\min }\right)=\min _{\alpha} f(\alpha)
$$

or

$$
\left(\frac{\partial f(\alpha)}{\partial \alpha}\right)_{\alpha=\alpha_{\min }}=0
$$

\section{b. 2nd iteration}

we substitute the angular distribution function $\eta(\theta)$ obtained in the first step for the expression for $h_{\nu \nu^{\prime}}$ into Eq.(19) to calculate $n_{\nu}$ in the second iterative step. Since $\eta(\theta)$ is independent of $\nu$ in the 1st iterative step, it follows from Eq.(19) that

$$
h_{\nu \nu^{\prime}}-h_{1 \nu^{\prime}}=0
$$


Substitution of Eq. $(\mathrm{H} 7)$ into Eq.(35) provides the expression for $n_{\nu}$ in the second iterative step

$$
n_{\nu}=C e^{-\nu y} e^{-\beta \Delta \mu_{\nu}^{(0)}}
$$

In this work, we chose

$$
\beta \Delta \mu^{(0)}=-z \ln \nu
$$

where $z$ is a parameter. Interestingly, the substitution of Eq. $(\mathrm{H} 9)$ into Eq. $(\mathrm{H} 8)$ provides an expression for the size distribution function $n_{\nu}$ in the asymmetric Schulz-Zimm form

$$
n_{\nu}=\frac{y^{z+1}}{\Gamma(z+1)} e^{-\nu y} \nu^{z},
$$

where $C=\frac{y^{z+1}}{\Gamma(z+1)}$ is a constant, which comes from the normalization condition in Eq.(2). According to Eq.(B7), the polydispersity parameter $z$ is related to the standard deviation $\sigma$. According to Eq.(B5), the parameter $y$ in Eq. $(\mathrm{H} 10)$ depends on the values $\langle\nu\rangle$ and $z$ only, i.e

$$
y=\frac{(z+1)}{<\nu>}
$$

Some other properties of Schulz's distribution are included in Appendix B.

Finally, the substitution of $n_{\nu}$ from Eq. $(\mathrm{H} 10)$ into Eq.(41) gives an equation to find $\eta_{\nu}(\theta)$. We note that getting the solution of this equation is not an easy task. Instead of solving Eq.(41), we introduce the following parametrization for polydisperse angular distribution function [19]

$$
\eta_{\nu}(\theta)=\frac{\alpha_{\nu}}{4 \pi} e^{-\frac{\alpha_{\nu}}{2} \theta^{2}}
$$

which generalizes the monodisperse trial function given in Eq. $(\mathrm{H} 4)$ to the polydisperse case. As a result, the Gaussian parameter $\alpha_{\nu}$ in Eq. $(\mathrm{H} 12)$ is a function of the size of filament $\nu$.

We assume that the polydispersity of the system is weak to avoid lengthy expressions and costly calculations. It is reasonable to assume that the distribution $\eta_{\nu}(\theta)$ becomes sharper for larger size of filaments $\nu$, i.e. $\alpha_{\nu}$ increases with increase of $\nu$. Consequently, we use the following approximation for the Gaussian parameter $\alpha_{\nu}[19]$ 


$$
\alpha_{\nu}=\alpha\left(1+\frac{4}{3} \frac{\Delta \nu}{<\nu>}\right.
$$

where $\Delta \nu=\nu-\langle\nu\rangle$ and the ratio $\frac{\Delta \nu}{\langle\nu\rangle}<<1$ represents a small parameter. In the monodisperse limit, i.e. for $\Delta \nu \rightarrow 0$, it follows from Eq. $(\mathrm{H} 13)$ that $\alpha_{\nu} \rightarrow \alpha$, i.e. Eq. $(\mathrm{H} 12)$ becomes Eq. $(\mathrm{H} 4)$. Again, the single unknown parameter $\alpha$ in Eq. $(\mathrm{H} 13)$ can be found as the numerical solution of Eq.(H5). 


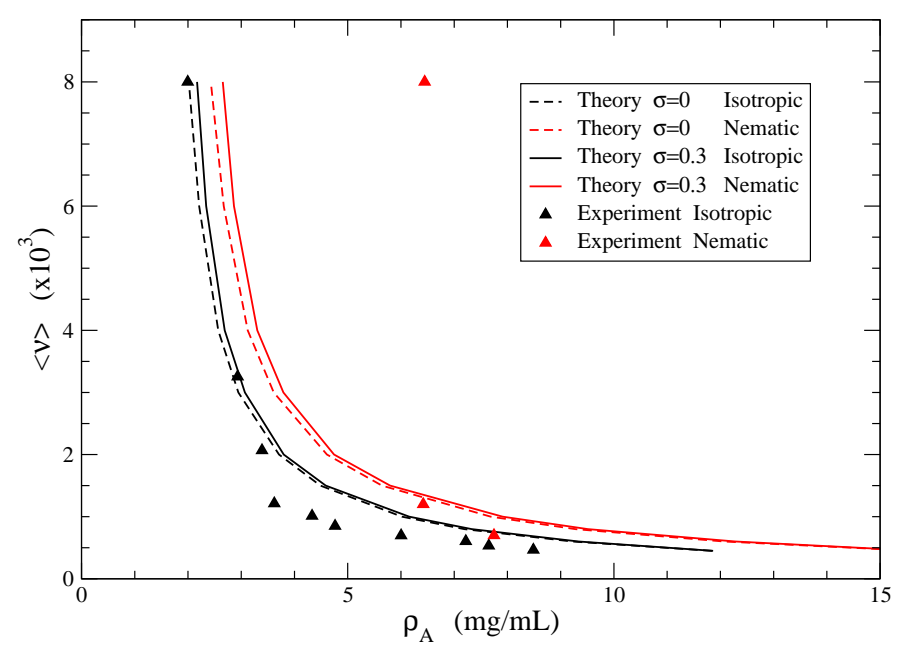

Figure 1: The isotropic-nematic phase diagram in term of coexisting densities of actin $\rho_{A}$ for different average length of filaments $\langle\nu\rangle$ for two values of normalized standard deviation $\sigma=0$ and $\sigma=0.3$. The persistence length $P=18 \mu \mathrm{m}$, the electrolyte concentration $c_{e}=0.1 M$. The experimental data are from Ref.[15]. 


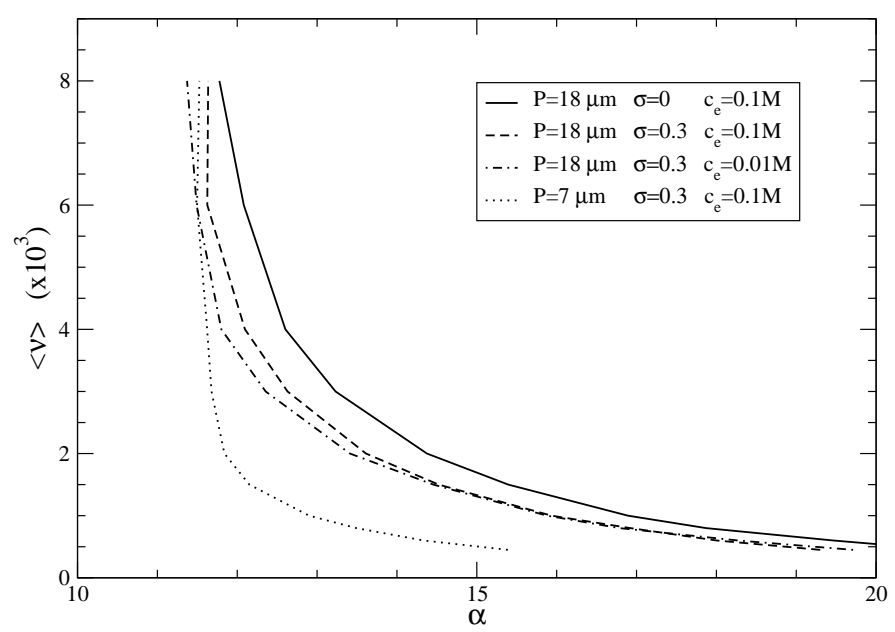

Figure 2: The Gaussian parameter $\alpha$ of nematic phase at equilibrium for different average length of filaments $\langle\nu\rangle$ for different sets of values of electrolyte concentration $c_{e}$, persistence length $P=18 \mu m$ and the normalized standard deviation $\sigma=0.3$. These sets of values correspond to the ones at Figs. 1,4,6.

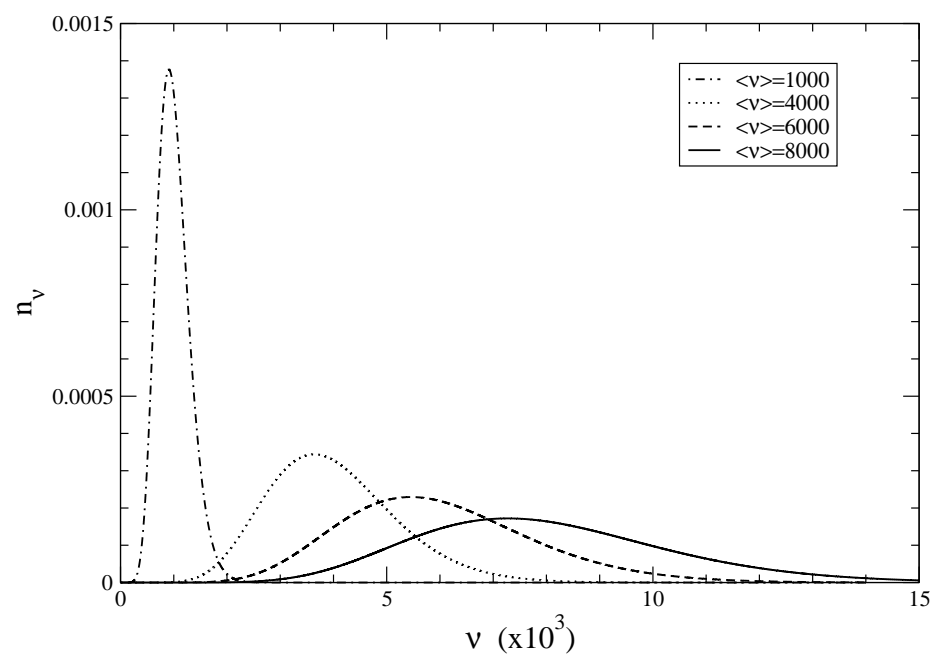

Figure 3: Schulz's distribution function $n_{\nu}$ vs the filament size $\nu$ for different average sizes $\langle\nu\rangle$. The normalized standard deviation $\sigma=0.3(z=10)$. 


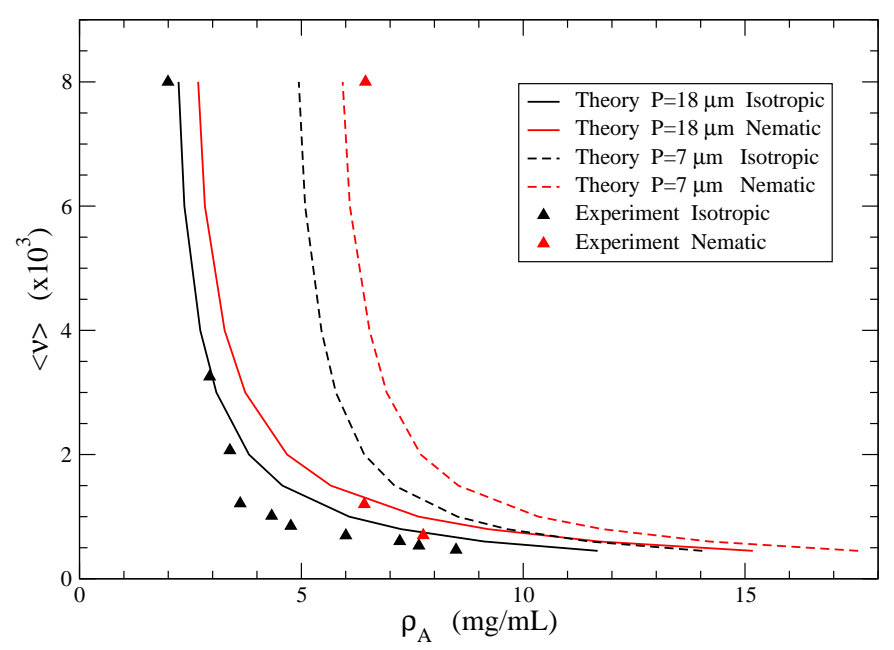

Figure 4: The isotropic-nematic phase diagram in term of coexisting densities of actin $\rho_{A}$ for different average length of filaments $\langle\nu\rangle$ for two values of persistence length $P=7 \mu m$ and $P=18 \mu m$. The normalized standard deviation $\sigma=0.3$, the electrolyte concentration $c_{e}=0.1 M$. The experimental data are from Ref.[15].

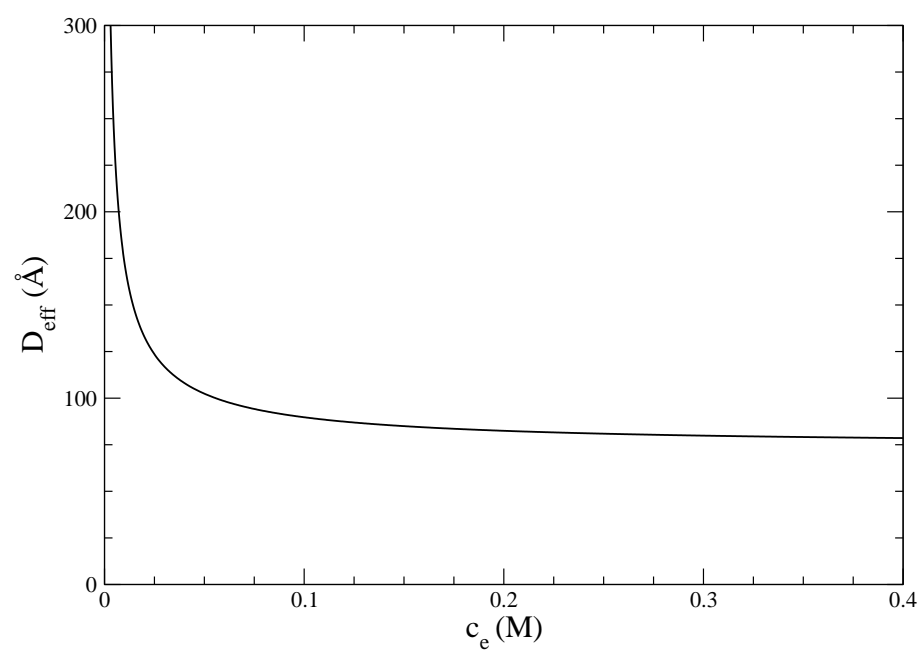

Figure 5: The effective diameter $D_{e f f}$ vs the concentration of monovalent ions $c_{e}$. 


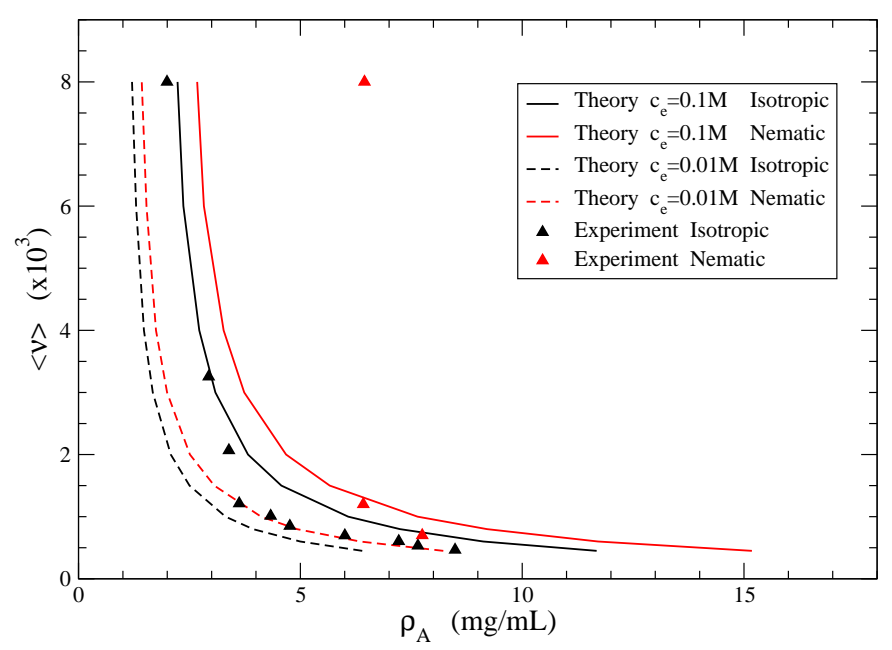

Figure 6: The isotropic-nematic phase diagram in term of coexisting densities of actin $\rho_{A}$ for different average length of filaments $\langle\nu\rangle$ for two values of electrolyte concentration $c_{e}=0.1 M$ and $c_{e}=0.01 M$. The persistence length $P=18 \mu m$, the normalized standard deviation $\sigma=0.3$. The experimental data are from Ref.[15]. 